\title{
Scale-free behavior in hailstone sequences generated by the Collatz map
}

\author{
M. G. E. da Luz $\odot,{ }^{1,{ }^{*}}$ D. M. G. dos Santos $\odot,{ }^{2}$ E. P. Raposo, ${ }^{3}$ and G. M. Viswanathan ${ }^{2,4}$ \\ ${ }^{1}$ Departamento de Física, Universidade Federal do Paraná, Curitiba, Paraná 81531-980, Brazil \\ ${ }^{2}$ Departamento de Física, Universidade Federal do Rio Grande do Norte, Natal, \\ Rio Grande do Norte 59078-970, Brazil \\ ${ }^{3}$ Laboratório de Física Teórica e Computacional, Departamento de Física, Universidade Federal de Pernambuco, Recife, \\ Pernambuco 50670-901, Brazil \\ ${ }^{4}$ National Institute of Science and Technology of Complex Systems, Universidade Federal do Rio Grande do Norte, Natal, \\ Rio Grande do Norte 59078-970, Brazil
}

(Received 1 November 2019; accepted 27 December 2020; published 22 January 2021)

\begin{abstract}
The Collatz conjecture, perhaps the most elementary unsolved problem in mathematics, claims that for all positive integers $n$, the map $n \mapsto n / 2$ ( $n$ even) and $n \mapsto 3 n+1$ ( $n$ odd) reaches 1 after a finite number of iterations. We examine the Collatz map's orbits, known as hailstone sequences, to determine whether or not they exhibit scale-invariant behavior, in analogy with certain processes observed in real physical systems. We develop an efficient way to generate orbits for extremely large $n$ (e.g., higher than $n \sim 10^{3000}$ ), allowing us to statistically analyze very long sequences. We find strong evidence of a scale-free power law for the Collatz map. We analytically derive the scaling exponents, displaying excellent agreement with the numerical estimations. The scale-free sequences seen in the Collatz dynamics are consistent with geometric Brownian motion with drift, which is compatible with the validity of the Collatz conjecture. Our results lead to another conjecture (conceivably testable through direct, nonetheless very time consuming, numerical simulations): Given an initial $n$, the average number of iterations needed to reach 1 is proportional, to lowest order, to $\log [n]$ (basis 10 ).
\end{abstract}

DOI: 10.1103/PhysRevResearch.3.013073

\section{INTRODUCTION}

Complexity, already a more than 70-year-old notion [1], has become an important framework for the proper understanding of a huge number of natural (and artificial) systems [2]. However, unlike many concepts in physics, its definition is not always concise or precise [3]. In fact, complexity is based on a relatively large set of features [2,3], e.g., similarity (or not) of constituents, nonlinearity, feedback responses (interdependence), self-organization, robustness to perturbations, critical and emergent properties, etc., which, when occurring more or less concomitantly, can characterize complex behavior. Due to this diversity of factors, it can be more useful to think about distinct groups of problems as possessing different degrees of complexity, forming hierarchical families of processes [4-6].

Nonetheless, one of the key features of many complex systems relates to hierarchical organization or self-similar structure [5,7]. The mathematical signature of self-similarity, and more generally self-affinity, is the presence of a power law for some relevant quantity describing the dynamics. Functions with asymptotic power-law decay are characterized by the

\footnotetext{
*luz@ fisica.ufpr.br

Published by the American Physical Society under the terms of the Creative Commons Attribution 4.0 International license. Further distribution of this work must maintain attribution to the author(s) and the published article's title, journal citation, and DOI.
}

absence of typical scales, in the sense that for $f(x)=x^{-\mu}$ one has $f(\alpha x)=\alpha^{-\mu} f(x)$ for any dilation $\alpha \neq 0$. Often power laws arise from the exact cancellation of two exponentials when a tunable parameter assumes a special critical value [8]. At this critical point, the system becomes scale-free, so the correlation lengths diverge. This is exactly the case for continuous phase transitions, as in the classic two-dimensional ferromagnetic Ising model [9].

Still, criticality in this technical sense is not a necessary or even a sufficient condition for a system as a whole to become scale-free. For instance, fractals (structures not necessarily associated with critical phenomena) have scale-free properties. Another example is weak chaos, in which the Lyapunov exponent is zero. Thus, initially close orbits separate not exponentially but rather with power-law growth. Yet another example concerns random walks with long-range power-law correlations, such as Lévy walks and flights. The randomwalk propagator for a Lévy flight has power-law tails, whereas for a Lévy walk the propagator dynamically evolves these power-law tails (because of aging). None of these cases are directly associated with criticality, but they share with critical phenomena certain scale-free properties.

Also related to scale-free behavior is the idea of selforganized criticality (SOC) $[10,11]$. Although not unconditionally implying complexity [12-14], SOC proposes both emergent organization and criticality as potential identifying trends of complex behavior [15-18]. Contrasting with continuous phase transitions [8], in SOC the dynamics itself drives the system towards a critical scale-free distribution of a relevant parameter. In particular, inherent fluctuations resulting 
in avalanchelike patterns of activity [19] are a key mechanism maintaining the system at a critical state. Since the paradigmatic sandpile model [10] (but see [20,21]), several realistic systems [22] have been investigated from the perspective of SOC. Complying with the view of distinct levels of complexity, few systems have been proposed as minimal illustrations of SOC. Examples range from simple deterministic [23] and stochastic [24] models to purely mathematical [25,26] constructions.

Motivated by the above discussion, in this work we look at how scale-free behavior can emerge in a straightforward purely mathematical evolution, hence having some, but far from all, the typical aspects associated with physical criticality $[4,9]$. In other words, we will address scale-free behavior and the existence of potential scale-invariant evolution in a very basic deterministic rule of the kind $x_{t+1}=f\left(x_{t}\right)$. Such a type of analysis certainly bears the fundamental question of how elementary a dynamics can be while still leading to certain emergent features of complexity (here self-similarity) [27]. Indeed, efforts have been put forward in methods to identify power-law behavior and their stability in dynamical systems [28,29]. We should emphasize that this is not just an academic curiosity. For instance, since bona fide complexity can normally emerge from deterministic evolution taking place in innate scale-free networks [30,31], eventually it could justify the apparent ubiquity of complex phenomena in nature $[2,4,6,9]$. However, this explanation may not be so direct as realistic true scale-free networks might not be as common as previously thought [32].

The most elemental rule in the form $x_{t+1}=f\left(x_{t}\right)$ should involve only positive integers [25]. We consider then the mapping of the Collatz conjecture [33]. This approximately 80 -year-old open problem is considered by many to be one of the hardest in all of mathematics, allegedly making Erdős comment that "mathematics may not be ready for such problems." Since this "evolution law" is taken from number theory [34], there are no experimental limitations or conceptual difficulties related to the underlying physics [13]. We report findings of scale-free features in the Collatz dynamics as defined below, which in many aspects is even more straightforward than the integer-based model in [25].

The work is organized as follows. In Sec. II we first review the Collatz conjecture and its corresponding map $f$. Second, we explain our method of analysis to identify scale-free behavior for proper orbits generated by $f$. In Sec. III we present our main findings, considering a few orbits in detail as well as addressing statistical properties of a collection of orbits. In Sec. IV we conclude with several remarks and also argue that our results are consistent with the validity of the Collatz conjecture.

\section{COLLATZ MAP DYNAMICS}

Certain important (arguably true) facts are simple to state but extremely difficult to prove. The Collatz conjecture [35] surely belongs to such a class of renowned hard open problems. Let $n \in \mathbb{N}^{+}$be a positive integer and $f: \mathbb{N}^{+} \rightarrow \mathbb{N}^{+}$a function, where

$$
f(n)= \begin{cases}n / 2, & n \text { even } \\ 3 n+1, & n \text { odd }\end{cases}
$$

The Collatz dynamics is generated by iterating the map $n \mapsto$ $f(n)$. Successive applications produce a sequence of integers $\left\{n_{0}, n_{1}, n_{2}, n_{3}, \ldots\right\}$ (with $n_{0}$ the initially chosen integer to start off the sequence). Each successor is half or approximately triple the value of its predecessor. From the notation $f^{(0)}\left(n_{0}\right)=n_{0}, f^{(1)}\left(n_{0}\right)=f\left(n_{0}\right)=n_{1}, f^{(2)}\left(n_{0}\right)=f\left(f\left(n_{0}\right)\right)=$ $n_{2}, f^{(3)}\left(n_{0}\right)=f\left(f\left(f\left(n_{0}\right)\right)\right)=n_{3}$, etc., we have that the $S$ th Collatz step is given by $f^{(S)}\left(n_{0}\right)$. We denote by $\left\{f^{(S)}\right\}$ the sequence of the above numbers up to $n_{S}$. The $\left\{f^{(S)}\right\}$ is known as a hailstone sequence because of the multiple ascents and descents that hailstones go through during their formation [36].

The Collatz conjecture states the following [33,35,37]: For every positive integer $n_{0}$, the hailstone sequence generated by $n \mapsto f(n)$ will necessarily reach the number 1 . In other words, for all $n_{0} \in \mathbb{N}^{+}$there exists a finite $T$ (which, however, can be very large) such that under $f, \mathcal{O}\left(n_{0}\right) \equiv\left\{n_{0}, n_{1}, n_{2}, \ldots, n_{T}=\right.$ 1) holds or similarly $f^{(T)}\left(n_{0}\right)=1$ (note that from $n_{T}=1$, subsequently $\left.\left\{\ldots, n_{T}=1,4,2,1,4,2,1, \ldots\right\}\right)$. We call any sequence $\mathcal{O}$ of this form an orbit whose period is $T$. The present record in testing positively the Collatz conjecture is for all $n_{0}$ up to $5 \times 2^{60}>5.764 \times 10^{18}$ [38] with the highest $n_{0}$ already tested being $2^{100000}-1$ [39]. We also should mention that recently Tao has shown that "almost all orbits of the Collatz map attain almost bounded values" [40].

Following the aforementioned philosophy of unveiling minimal models displaying certain universal ingredients, recurrent in actual physical processes, below we look for power-law characteristics in the Collatz orbits.

There is a major feature of the Collatz dynamics which must be taken into account. While looking for power laws, long periods $T$ are required for a proper statistical analysis, demanding very large initial $n_{0}$ 's. However, even then, a small fraction of the resulting orbits may not be typical. A few may be very short and others extremely long but with a repetitive trivial succession of ascents and descents in their hailstone sequence. Moreover, the majority of sequences (hereafter called typical orbits) usually need to pass through intermediate integers that can be considerably larger than the initial number. Thus, we have developed a method for representing large positive integers, which is particularly suitable for the Collatz map. Also, through numerical tests with such a protocol, we have determined typical initial $n_{0}$ 's, potentially leading to scale-free behavior in the orbits $\mathcal{O}\left(n_{0}\right)$. We briefly describe our approach next (for more details see Appendix A).

We must work with gigantic natural numbers $n$, to which the Collatz map $f$ must be applied. Then a proper decomposition considerably helps to find good candidates $n_{0}$ for the scale-free orbits, also being very handy for the computational implementation of $f$. Our decomposition method is as follows. Any $n \in \mathbb{N}^{+}$can always be represented in a unique way by

$$
\begin{aligned}
n & =2^{m_{1}}+2^{m_{1}+m_{2}}+2^{m_{1}+m_{2}+m_{3}}+\cdots+2^{m_{1}+m_{2}+m_{3}+\cdots+m_{r}} \\
& \equiv\left(m_{1}, m_{2}, m_{3}, \ldots, m_{r-2}, m_{r-1}, m_{r}\right) .
\end{aligned}
$$

The uniqueness is guaranteed by imposing certain conditions on the $m_{i}$ 's (Appendix A). As an illustration, we have

$$
\begin{aligned}
& n=7=2^{0}+2^{0+1}+2^{0+1+1} \equiv(0,1,1), \\
& n=26=2^{1}+2^{1+2}+2^{1+2+1} \equiv(1,2,1) .
\end{aligned}
$$



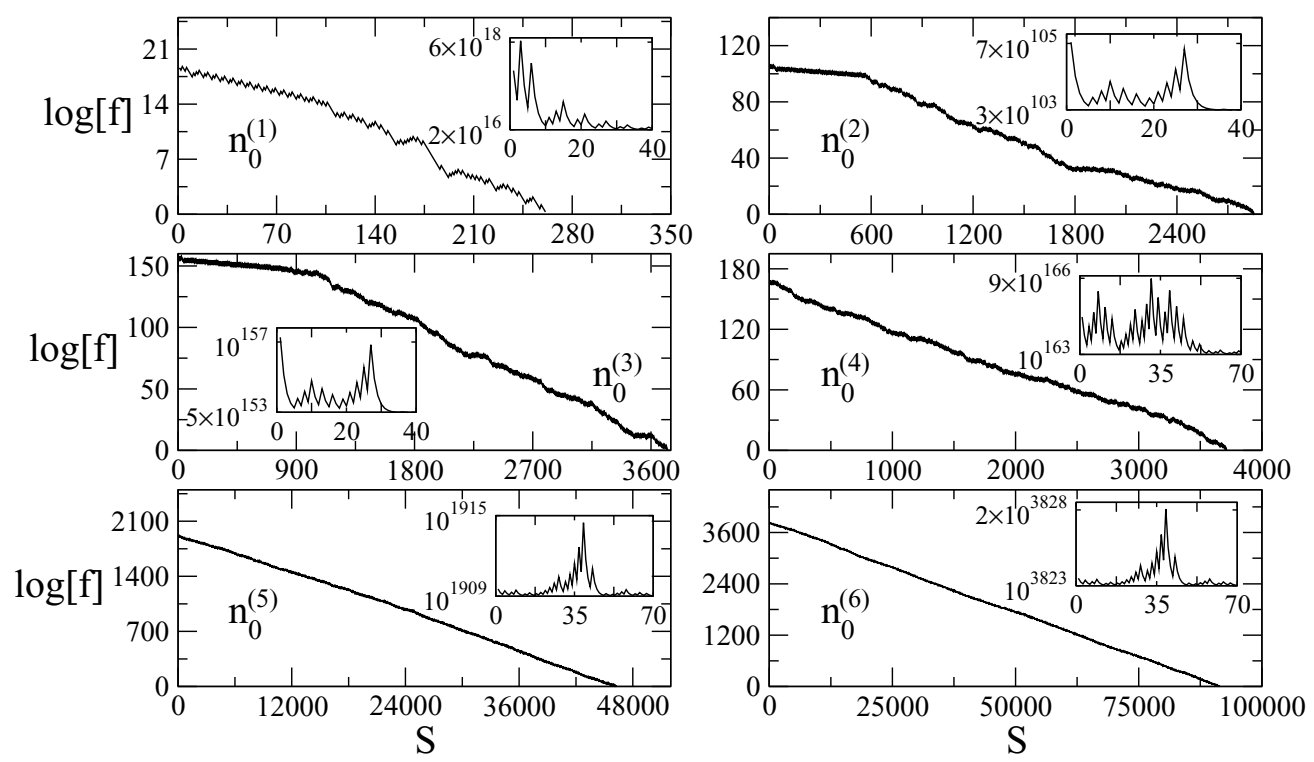

FIG. 1. Semilogarithmic plots of $\log [f] \times S$, showing the hailstone sequences $\left\{f^{(S)}\left(n_{0}\right)\right\}$ (until $S=T$ ) for the six $n_{0}$ values considered in details in this work. Logarithms are in base 10. The insets display a close-up of the first few steps of the sequences in a linear scale. The hailstones $\left\{f^{(S)}\left(n_{0}\right)\right\}$ resemble a 1D RW with drift towards an absorbing boundary at the termination value 1 .

Also, for any $n$ there is a simple and rapid algorithm to derive its exponent set $\left(m_{1}, m_{2}, \ldots, m_{r}\right)$ (Appendix A).

Using this construction, we have written a code to generate the hailstone sequences of the Collatz map, assuming properly chosen $n_{0}$ that can be as large as $10^{1000}$ (or even larger). The resulting orbits consequently can have extremely long $T$ 's. For these orbits $\mathcal{O}\left(n_{0}\right)$ we have looked for scale-free behavior. In particular, we have analyzed the sizes of the jumps and their frequency. Note that although in this work we show only six sequences in greater detail for their dynamics (for statistical analysis we consider a set of 50 sequences), we have looked at many more to check that our findings are in fact general.

\section{RESULTS}

We have performed an exhaustive investigation and here we present just a few representative examples. Thus, we discuss only six values for $n_{0}$, labeled $n_{0}^{(1)}<$ $n_{0}^{(2)}<n_{0}^{(3)}<n_{0}^{(4)}<n_{0}^{(5)}<n_{0}^{(6)}$. Their total numbers of digits (in the usual base-10 number system) are, respectively, $19,106,157,167,1914,3827$. For instance, for $n_{0}^{(1)}$,

$$
\begin{aligned}
n_{0}^{(1)}= & (0,1,2,3,1,2,3,1,2,3,1,2,3,1,2,3,1,2,3,1,2, \\
& 3,1,2,3,1,2,3,1,2,3) \\
= & 1,354,225,259,379,471,051 .
\end{aligned}
$$

Using the representation in Eq. (2), $n_{0}^{(4)}=(0,3,5,7,77$, $3,5,7,77,3,5,7,77,3,5,7,77,3,5,7,77,3,5,7,77)$, and for $n_{0}^{(2)}, n_{0}^{(3)}, n_{0}^{(5)}$, and $n_{0}^{(6)}$ the number of exponents are $r=106,157,867$, and 1773, respectively. Also, $m_{1}=0$ for all the $n_{0}$ 's and for the remaining $r-1$ exponents (from $m_{2}$ to $m_{r}$ ) we have a repeated sequence of $2,3,5$ for $n_{0}^{(2)}$ and for $n_{0}^{(3)}$; a repeated sequence of 5,7,11, unless for $m_{104}-m_{114}, m_{319}-m_{329}, m_{534}-m_{544}, m_{749}-m_{759}$, and $m_{862}-m_{867}$ (all equal to 2 ) for $n_{0}^{(5)}$; and a repeated sequence of $5,7,11$, unless for $m_{104}-m_{114}, m_{319}-m_{329}, m_{534}-m_{544}$, $m_{749}-m_{759}, m_{862}-m_{867}, m_{970}-m_{980}, m_{1185}-m_{1195}, m_{1400}-m_{1410}$, $m_{1615}-m_{1625}$, and $m_{1728}-m_{1733}$ (all equal to 2) for $n_{0}^{(6)}$. For completeness, the full coefficients list can be seen in Appendix A.

Since these numbers are very large, it is convenient to use a logarithmic (base-10) scale to plot the relevant quantities graphically. We show histograms on a double-logarithmic scale, so we have employed the logarithmic binning method for better visualization.

An important (but still unproven) hypothesis associated with the Collatz conjecture is that for $n_{0}$ chosen uniformly at random, on average the decay of $\left\{f^{(S)}\left(n_{0}\right)\right\}$ also resembles a fairly random sequence [41,42]. Hence, any measure based on the drops (to reach the number 1) should be subjected to fluctuations. In fact, in a semilogarithmic plot we see from Fig. 1 (for all six values of $n_{0}$ ) that typically the hailstone sequences look similar to a one-dimensional (1D) (geometric) random walk (RW) with a drift [43-46] towards an absorbing boundary (at the termination value 1). Thus, if indeed the Collatz dynamics could be mapped onto a biased 1D RW, the Collatz conjecture would be readily demonstrated (conceivably by induction) from the famous directed graph structure of the Collatz orbits [33].

Despite the similarity to a 1D drifted RW [43,45], shown in Fig. 1, it is difficult to observe relevant differences between small and large fluctuations. However, better insight into the general trend of the hailstone Collatz sequences is obtained by constructing a simple normalized histogram of $\left\{f^{(S)}\left(n_{0}\right)\right\}$, and thus generating the distribution $D(f)$. The resulting curves in $\log -\log$ plots are depicted in Fig. 2. They are consistent with straight lines, indicating a power law for $D(f)$ in the form

$$
D(f) \sim f^{-\alpha},
$$

with $\alpha=1.0$ regardless of $n_{0}$. We can interpret $\alpha$ as the critical exponent reflecting the effect of fluctuations (up and down) of the dynamics. It is worth mentioning that a 

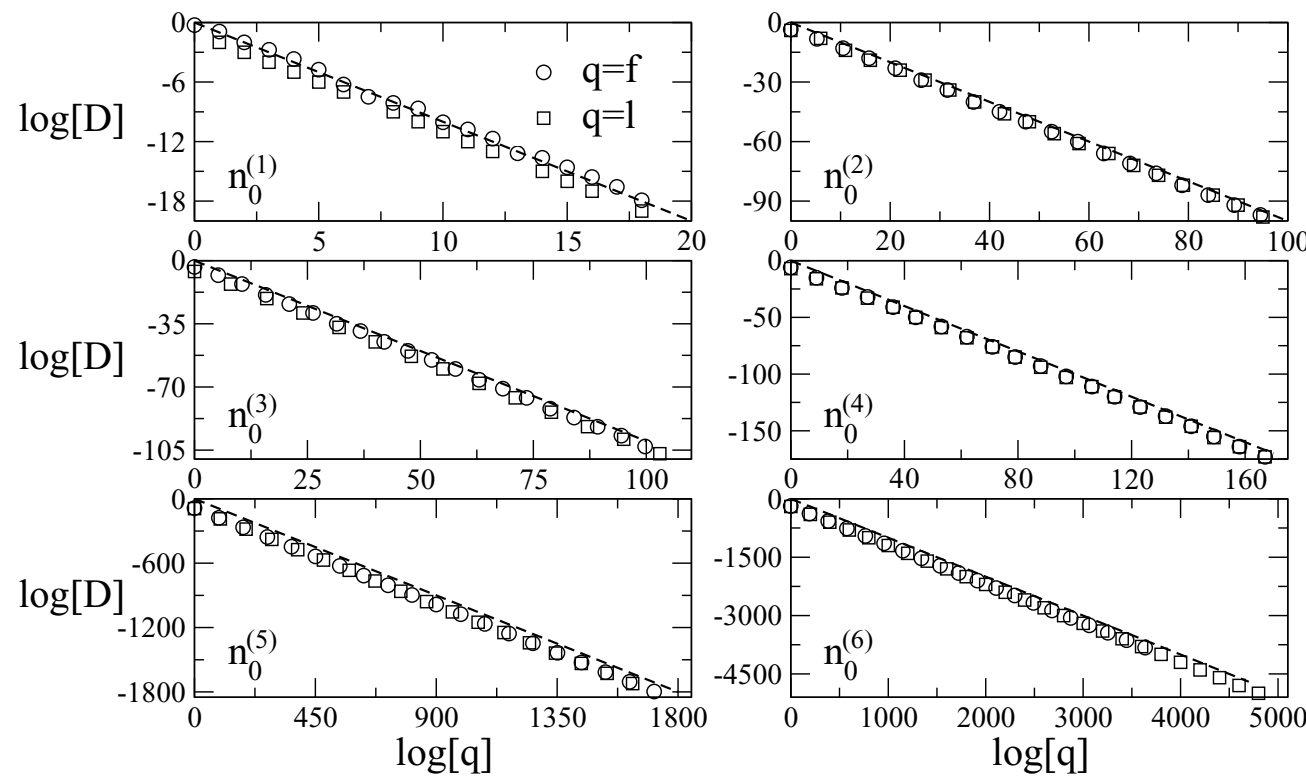

FIG. 2. The $\log$-log plots $\log [D] \times \log [q]$ of normalized histograms for the six $n_{0}$ values considered in this work. Logarithms are in base 10. The circles represent the distribution $D(f)$ for the hailstone sequence values $f$. The squares represent the distribution $D(l)$ of the avalanche lengths $l$ as defined in the main text. In both cases $D(q) \sim q^{-1}$ for all $n_{0}$.

$D(f) \sim 1 / f$ signal is commonly associated with selforganization in different systems [10,47]. For example, it arises in concrete instances where the physical system undergoes a drift to an absorbing state (e.g., due to dissipation) [48].

Another important characterization of scale-free behavior is the distribution of magnitudes of a certain variable of interest $v$, resulting from the system's time evolution. The famous avalanches in the theoretical sandpile model [10] in fact mean that $v$ can display very large fluctuations, much more frequent than expected from a usual normal distribution. In other words, $v$ should obey a power-law-like distribution. For the Collatz map, we also analyze the sizes (or lengths) $l$ of the avalanches. We should note that the word "avalanche" in our context is just an analogy; it is not supposed to bear the exact same meaning as that in SOC. In fact, an appropriate way in the present context to define the quantity $l$ is as follows. Consider all the distinct stretches of orbits $\mathcal{O}\left(n_{0}\right)$ which fall without interruption. Each one of these stretches corresponds to a (small or big) avalanche. We suppose the $j$ th stretch sequence of $\mathcal{O}\left(n_{0}\right)$, having the first and last terms $n_{j, i}$ and $n_{j, f}$, respectively. We define then $l_{j}=n_{j, i}-n_{j, f}$. For example, $\mathcal{O}\left(n_{0}=48\right)=\{48,24,12,6,3,10,5,16,8,4,2,1\}$, leading to three avalanches: from 48 to 3 (with $l_{1}=48-3=45$ ), next from 10 to 5 (with $l_{2}=10-5=5$ ), and finally from 16 to 1 (with $l_{3}=16-1=15$ ). For a long enough orbit, we can calculate the distribution of $D(l)$, which for a scale-free process should also follow a power law. In Fig. 2 we show $D(l)$ for all six $n_{0}$. The numerical analysis fits perfectly well the power-law form $D(l) \sim l^{-\alpha_{a v}}$, again for the critical exponent $\alpha_{a v}=1.0$. The numerical coincidence of $\alpha$ in Eq. (4) with $\alpha_{a v}$ is addressed below.

For our next numerical analysis, it is useful to define the Collatz map in a slightly different way. Recall that if $n$ is odd, then $3 n+1$ is even, and so necessarily $n \mapsto 3 n+1 \mapsto$ $(3 n+1) / 2$. Thus, without loss of generality we can redefine the Collatz dynamics as

$$
\tilde{f}(n)= \begin{cases}n / 2, & n \text { even } \\ (3 n+1) / 2=n+(n+1) / 2, & n \text { odd. }\end{cases}
$$

For these $\tilde{f}$ sequences we can ask about the time duration (in steps) of stretches corresponding to either successive increases or successive decreases of the corresponding $n_{S}$ 's. For instance, for $\left\{\tilde{f}^{(S)}(48)\right\}=\{48,24,12,6,3,5,8,4,2,1\}$, first we find a decrease during four steps $(48 \rightarrow 24 \rightarrow 12 \rightarrow$ $6 \rightarrow 3)$, then an increase during two steps $(3 \rightarrow 5 \rightarrow 8)$, and a final decrease during three steps $(8 \rightarrow 4 \rightarrow 2 \rightarrow 1)$. Thus, for the $j$ th stretch (which now can be either an up or a down sequence), we define

$$
t_{j}=\log \left[\frac{n_{j, i}}{n_{j, f}}\right] .
$$

Here $t_{j}>0\left(t_{j}<0\right)$ if we have a down (an up) avalanche, as previously defined. The logarithm in Eq. (6) is justified because for a down avalanche where $n_{j, i}=2^{m} n_{n, f}$, we have that $t_{j} \approx 0.3 m$ is proportional to the avalanche's number of steps $m$ (we do not use $\log _{2}$, for which $t_{j}$ would be exactly $m$, for uniformity with the previous analysis). In Fig. 3 we show the $t$ 's for the successive up and down stretches $j$ for six $n_{0}$ 's. The insets of Fig. 3 display the corresponding $D(t)$ 's, but only for the down avalanches.

What could be the mechanism that underpins the observed scale-free behavior? Observe in Fig. 1 that the logarithm of the sequence appears to be of uniform velocity with a noise term added. The simplest stochastic process with these properties is geometric Brownian motion. Indeed, the overall picture emerging from the analysis of our examples so far is that of geometric Brownian motion (GBM) [43,44]. However, then a pertinent issue concerns the robustness of these findings, namely, their statistical significance. Before we address this question, we stress an important distinction between pure mathematics and physics, appropriate in the present study. In 

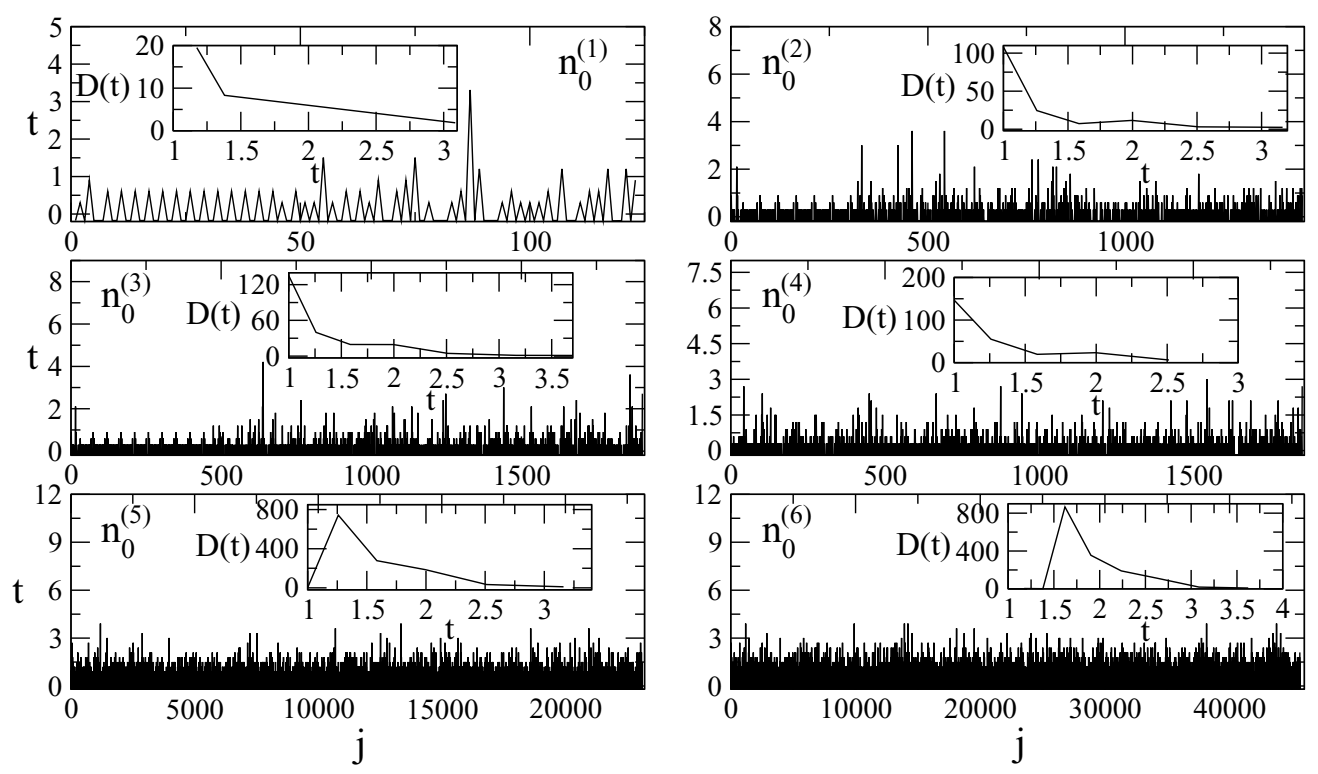

FIG. 3. Quantity $t_{j}$, defined in Eq. (6), for the up and down avalanches $j$. The insets show the distributions $D(t)$ of the down avalanches. Since the number of steps (or time duration) $m$ of a down avalanche is roughly $m=t / 0.3$, we see that although the avalanches duration distribution is not exactly a power law, it is also not a distribution with a sharp mean.

pure mathematics a probability of $100 \%$ is not necessarily as meaningful as it is in physics. For example, since the rationals are a countable set, they have a measure of zero in the reals and hence their density is also zero in $\mathbb{R}$. Thus, although the probability to pick uniformly an irrational in the line is $100 \%$, this certainly does not imply that there are no rationals. Following the same reasoning, it is not enough or even meaningful to say that the Collatz conjecture is valid for $100 \%$ of all initial conditions or that $100 \%$ of the zeros of the Riemann $\zeta$ function analyzed lie on the critical line. Even a small exceptional set of measure zero is enough to invalidate these hypotheses. A single counterexample constitutes disproof, in which case questions of statistical significance are not relevant.

On the other hand, considered as a dynamical system, it is still fair to ask about how confident we are about the claim of GBM for an important fraction of arbitrarily chosen very large $n_{0}$ 's. Therefore, we have performed an elementary self-consistency check as follows. Our hypothesis is that the observed power-law scaling is a consequence of the GBM. Of course, power-law scaling could have other causes, so in order to test for GBM we should focus not on the power-law scaling or the exponent $\alpha$, but rather on a quantity that is directly associated with the GBM. For arbitrary $n_{0}$, let $\gamma$ be the exponent defined according to $f^{(S)}\left(n_{0}\right) \sim n_{0} 10^{-\gamma S}$ for the drift in sequences of the type observed in Fig. 1. Assuming GBM, this exponent should fairly carry over (as discussed below) the distinct $\left\{f^{(S)}\left(n_{0}\right)\right\}$. Thus, we have generated 50 sequences with $1 \times 10^{13}<n_{0}<2 \times 10^{13}$ randomly chosen. Such an $n_{0}$ interval is already adequate for statistical analysis yet keeping the numerical work cost low. In Table I we list the $n_{0}$ 's as well as the estimated $\gamma$ for each sequence (taking the initial and ending points to obtain the exponential fit; thus no regression, least squares, spline, etc., have been used). For this sample of 50 exponents, the mean is $\bar{\gamma}=0.04663$. The standard deviation is $\sigma=0.0112$.
If we really have GBM, then if we nonrandomly split these 50 sequences, say, into two sets of 25 sequences (splitting into more sets, i.e., three, four, etc., would surely require more than 50 initial $n_{0}$ 's), a two-sample $t$-test will immediately be able to reveal an estimate of the probability of whether the $\gamma$ were generated by the same process. Obviously, a random division is not expected to show differences in statistical properties. To maximize systematic bias and check for the worst case scenario, our two sets are composed of the 25 largest and 25 smallest $n_{0}$ 's in Table I. If the two sets have statistically significant differences in their $\gamma$, this difference would represent evidence against our hypothesis of GBM. Let the null hypothesis be that the two sets have the same mean. The alternate hypothesis is that the group means differ. We assume the standard significance level of 0.05 , often denoted by the symbol $\alpha$ (not to be mistaken for our exponent $\alpha$ ) in hypothesis testing theory. For the two-sample $t$-test we obtain a $p$-value of $p=0.19$ assuming the null hypothesis. Since $p=0.19>0.05$ is a relatively large $p$-value, we can conclude that there is no statistically significant difference between the group means. Recall that $p<0.05$ is frequently considered the gold standard of significance, so we find no relevant evidence for the alternative hypothesis. We can safely conclude that the evidence against GBM is not statistically significant (of course with the caveats related to mathematics versus physics mentioned above).

As a second, independent, test of the plausibility of GBM, we have calculated a theoretical value of $\gamma$ by assuming that we have a random process that depends only on the density of the even and odd numbers (see Appendix B). The theoretical estimate is $\gamma_{t}=0.041646$, which is nicely bracketed by $\bar{\gamma} \pm$ $\sigma=0.0466 \pm 0.0112$.

Some other relevant features can be inferred from Fig. 3. First, we do not find the same shape for all the $n_{0}$ 's. Second, the shapes do not indicate any kind of power law. However, by the same token, the $D(t)$ 's clearly do not represent wellconcentrated (e.g., normal) distributions for $t$. Notice also that 
TABLE I. Extra 50 values of $n_{0}^{(\#)}$ (randomly chosen) and the fittings for the $\gamma$ 's of the corresponding Collatz sequences.

\begin{tabular}{|c|c|c|c|}
\hline \# & $\left(m_{1}, \ldots, m_{r}\right)$ of $n_{0}^{(\#)}$ & $n_{0}^{(\#)}$ & $\gamma$ \\
\hline 07 & $3,1,2,2,5,1,2,2,1,3,2,1,5,3,7$ & 1109230707032 & 0.0412070 \\
\hline 08 & $4,1,2,2,7,2,2,3,2,1,2,2,3,7$ & 1109554168496 & 0.0451697 \\
\hline 09 & $4,1,2,2,5,2,2,2,3,2,1,2,2,3,7$ & 1109554184880 & 0.0451697 \\
\hline 10 & $4,1,2,5,2,2,2,2,3,2,1,2,2,3,7$ & 1109554188464 & 0.0451697 \\
\hline 11 & $3,1,2,2,5,2,2,2,1,3,2,1,2,2,3,7$ & 1109554536792 & 0.0593137 \\
\hline 12 & $5,3,2,2,3,1,2,5,2,5,2,3,5$ & 1139282384160 & 0.0593717 \\
\hline 13 & $1,5,7,2,2,4,7,2,2,3,5$ & 1139510779970 & 0.0430611 \\
\hline 14 & $1,3,5,7,1,2,5,7,1,3,5$ & 1140331315730 & 0.0350925 \\
\hline 15 & $1,3,5,2,5,1,2,5,7,1,3,5$ & 1140331317778 & 0.0593737 \\
\hline 16 & $3,1,2,2,5,1,2,2,1,3,2,1,5,3,2,5$ & 1143590445400 & 0.0476002 \\
\hline 17 & $3,1,2,2,5,1,4,4,2,1,3,2,3,2,5$ & 1143858291032 & 0.0513421 \\
\hline 18 & $5,1,2,5,1,4,4,2,1,3,2,3,2,5$ & 1143858291040 & 0.0513421 \\
\hline 19 & $3,1,2,2,5,1,5,3,2,1,3,2,3,2,5$ & 1143858553176 & 0.0350965 \\
\hline 20 & $1,1,5,7,2,2,3,2,2,2,5,2,1,5$ & 1155524804742 & 0.0351097 \\
\hline 21 & $1,3,5,3,2,3,2,2,5,2,7,2,3$ & 1271648637458 & 0.0301106 \\
\hline 22 & $1,3,5,7,2,1,2,3,2,2,7,2,3$ & 1271665590802 & 0.0252748 \\
\hline 23 & $1,3,5,7,2,1,4,3,2,2,5,2,3$ & 1272728846866 & 0.0334383 \\
\hline 24 & $5,3,2,2,3,1,2,7,5,2,3,2,3$ & 1276712949024 & 0.0611661 \\
\hline 25 & $5,3,2,2,3,5,3,2,5,2,3,2,3$ & 1276722058528 & 0.0440488 \\
\hline 26 & $5,3,2,2,3,1,5,2,2,5,2,3,2,3$ & 1276723172640 & 0.0611661 \\
\hline 27 & $5,3,2,2,5,5,2,2,5,2,2,2,3$ & 1282135954720 & 0.0412829 \\
\hline 28 & $1,3,5,1,3,3,2,2,2,2,5,2,3,1,2,3$ & 1291196900882 & 0.0559714 \\
\hline 29 & $5,3,2,7,2,2,3,2,1,2,2,3,1,2,3$ & 1291395400992 & 0.0366771 \\
\hline 30 & $1,5,3,1,3,3,2,5,2,2,5,2,1,2,3$ & 1292961654338 & 0.0315790 \\
\hline 31 & $5,3,2,3,2,1,4,3,7,2,2,1,2,3$ & 1293868442912 & 0.0395012 \\
\hline 32 & $1,3,5,1,3,5,1,3,5,1,3,5,1,3$ & 1308225185298 & 0.0498551 \\
\hline 33 & $1,2,3,1,2,3,1,2,6,3,1,2,3,1,2,3,1,3$ & 1317667910346 & 0.0612372 \\
\hline 34 & $1,2,3,1,2,3,1,2,3,1,2,3,1,2,3,1,2,3,1,3$ & 1317668696778 & 0.0656599 \\
\hline 35 & $1,3,3,1,3,3,2,2,2,1,3,3,1,3,3,2,2,2$ & 1462859286930 & 0.0275271 \\
\hline 36 & $1,3,1,7,3,7,2,2,2,1,3,7,1$ & 1654455832626 & 0.0388196 \\
\hline 37 & $1,3,7,1,3,7,2,2,2,1,3,7,1$ & 1654455834642 & 0.0388196 \\
\hline 38 & $1,2,3,3,3,1,2,6,1,2,3,1,2,3,6,1$ & 1659356885578 & 0.0461973 \\
\hline 39 & $3,1,2,3,3,1,2,6,1,2,3,1,2,3,6,1$ & 1659356885592 & 0.0608108 \\
\hline 40 & $1,2,3,1,2,3,1,2,6,1,2,3,1,2,3,6,1$ & 1659356885706 & 0.0461973 \\
\hline 41 & $1,2,3,1,1,1,3,1,2,6,1,2,3,1,2,3,6,1$ & 1659356885962 & 0.0357925 \\
\hline 42 & $2,1,3,1,1,1,3,1,2,6,1,2,3,1,2,3,6,1$ & 1659356885964 & 0.0357925 \\
\hline 43 & $5,1,2,3,3,1,6,1,2,3,1,2,3,6,1$ & 1659356891488 & 0.0608108 \\
\hline 44 & $5,1,2,3,3,1,2,2,2,2,1,3,1,2,3,6,1$ & 1659361741152 & 0.0461973 \\
\hline 45 & $1,2,3,5,1,3,6,1,2,2,2,1,3,2,5,1$ & 1671637801034 & 0.0388342 \\
\hline 46 & $1,3,2,5,1,3,6,1,2,2,2,1,3,2,5,1$ & 1671637801042 & 0.0388342 \\
\hline 47 & $1,3,2,5,1,3,6,1,2,3,1,1,3,2,5,1$ & 1671704909906 & 0.0476885 \\
\hline 48 & $1,3,7,1,3,7,2,2,2,1,3,2,3,2,1$ & 1809074657298 & 0.0353740 \\
\hline 49 & $1,3,7,1,3,2,3,2,2,2,2,1,3,2,3,2,1$ & 1809075836946 & 0.0724632 \\
\hline 50 & $5,1,1,2,2,4,3,3,1,2,2,2,1,3,2,3,2,1$ & 1809077013216 & 0.0474461 \\
\hline 51 & $1,3,7,1,3,3,3,1,2,2,2,1,3,2,3,2,1$ & 1809077016594 & 0.0472586 \\
\hline 52 & $1,3,2,5,1,3,3,3,1,2,2,2,1,3,2,3,2,1$ & 1809077016658 & 0.0472586 \\
\hline 53 & $5,1,2,6,1,4,4,1,3,1,2,3,2,2,2,1$ & 1831158202720 & 0.0421186 \\
\hline 54 & $5,1,2,6,1,2,2,2,2,1,3,1,2,3,2,2,2,1$ & 1831160430944 & 0.0672005 \\
\hline 55 & $5,1,2,6,1,4,4,1,1,1,1,1,2,3,2,2,2,1$ & 1831258866016 & 0.0572331 \\
\hline 56 & $5,1,2,6,1,4,4,2,1,3,2,3,2,1,2,1$ & 1875399721312 & 0.0626810 \\
\hline
\end{tabular}

in some cases we get long avalanches, with $m=12$ successively decreasing steps. These results indicate that although hailstone sequences are well approximated by GBM with drift, this identification is not the full story. Indeed, the actual dynamics is richer and more interesting.

For instance, for the increments (absolute values of the increments) of the hailstone sequences we have calculated the autocorrelation $C(\tau)[|C(\tau)|]$ as a function of the lag $\tau$. The calculations are depicted in Fig. 4 [we show $C$ and $|C|$ only for the orbit of $n_{0}^{(6)}$ (chosen since it has the longest sequence, so leading to a better statistics), but the other cases are qualitatively similar]. We see that the correlations exponentially decay with the lag $\tau$. Therefore, the orbits can be described more precisely as GBM with drift but with short- 


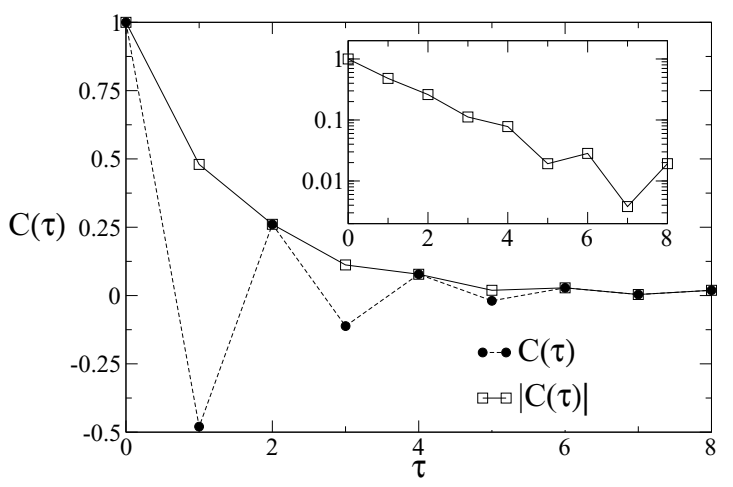

FIG. 4. Functions $C$ and $|C|$ versus the lag $\tau$ for the increments of the hailstone sequence of $n_{0}^{(6)}$ in Fig. 1. The inset shows $|C(\tau)|$ in semilogarithmic scale, evidencing an exponential decay. This behavior is inconsistent with Gaussian white ( $\delta$-correlated) noise. Such correlations are instead typical of one-step Markov chains. Hence, hailstone sequences are approximated by GBM, but only to low order since hailstone sequences contain short-range exponentially decaying correlations.

range correlations typical of one-step Markov processes. We note that in the initial intervals of $S$ in Fig. 1, the small oscillations and other deviations from (geometric) Brownian motion for $n_{0}^{(1)}$ and the plateaus for $n_{0}^{(2)}$ and $n_{0}^{(3)}$ might be due to these correlations. We finally observe that Brownian motion plus such short-range correlations cannot be described by usual diffusion or Fokker-Planck-like equations. Instead, it can can be well approximated by the telegrapher's equation as well as by the continuous-time RW formalism [49]. Nevertheless, upon renormalization of such correlated motion via coarse graining, in the long-time limit one always recovers the same diffusion properties and generalized Hurst exponents as for standard (i.e., uncorrelated) Brownian motion. This is so because short-range correlations cannot alter the stochastic dynamics long-time diffusion features.

We now analytically estimate the scaling exponents. A relevant question is why to expect the exact $\alpha$ values numerically found in our examples. A possible explanation is based on a simple hypothesis about the Collatz sequences, moreover bearing a possible strategy to prove the conjecture $[41,42]$ (see Sec. IV).

It seems that at least for some sets of Collatz orbits, the corresponding decaying sequences do resemble a stochastic processes, viz., geometric Brownian motion with drift. Such randomness (or pseudorandomness [37]) is more properly encoded into the logarithm of the step sizes rather than in their actual values (see, e.g., [50]). Thence, $\left\{\log \left[f^{(S)}\right]\right\}$ is a Brownian motion evolving towards the absorbing boundary at $\log \left[f^{(T)}\left(n_{0}\right)\right]=0$ such that $x_{S}=\log \left[f^{(S)}\left(n_{0}\right)\right]=\left\langle x_{S}\right\rangle+\xi_{S}$, with $\left\langle x_{S}\right\rangle$ approximately a linear decreasing function of $S$ and $\xi_{S}$ a stochastic (possibly Wiener-like) variable. Thus, the density of visited points $x_{S}$ is some constant $\rho_{S}$ and we can calculate the density of visited points $f^{(S)}$. Dropping $S$ in $x_{S}$ and $f^{(S)}$, note that $d x / d f=1 / f$ and

$$
\rho_{f} d f \approx \rho_{x} d x \Rightarrow \rho_{f} \approx \rho_{x} \frac{d x}{d f} \Rightarrow \rho_{f} \propto 1 / f .
$$

From Eq. (7), the points visited by the Collatz sequences are distributed not uniformly along the integers, but according to a density that scales inversely with the magnitude of the number. This gives us the $D(f)=1 / f$ distribution observed in Fig. 2.

For the power-law behavior of $D(l) \sim l^{-1}$ (Fig. 2), similar arguments (although a little trickier) apply. Assuming $n_{j, i}=2^{m} n_{j, f}$, we have $l_{j}=n_{j, i}-n_{j, f}=n_{j, i}\left(1-2^{-m}\right)$ and $\log \left[l_{j}\right]=\log \left[n_{j, i}\right]+\log \left[1-2^{-m}\right]$, which can be approximated as $\log \left[l_{j}\right] \approx \log \left[n_{j, i}\right]$ since most of the terms in our orbits are huge numbers and already for $m=2, \log [1-$ $\left.2^{-m}\right] \approx 0.125$ is comparatively very small. In this way, the distribution of $l_{j}\left[D(l) \sim l^{-\alpha_{a v}}\right]$ is essentially that of $n_{j, i}$. However, the $n_{j, i}$ 's are the onsets of all the down cascades. Thus, the distribution of the whole hailstone sequence $\left\{f^{(S)}\right\}$ should be dominated by the power-law behavior of $\left\{n_{j, i}\right\}$. In other words, the distribution of $\left\{n_{j, i}\right\}$ must have the same exponent $\alpha$ of $D(f) \sim f^{-\alpha}$. Consequently, $\alpha_{a v}=\alpha$.

\section{CONCLUSION}

We conclude the present work with a few general remarks and speculation on eventual implications of our findings.

First, one of the most exciting consequences of our study is that our results allow us to make a numerically testable theoretical prediction. Assuming that the Collatz dynamics is really a type of geometric Brownian motion with drift towards an absorbing lower boundary, we can estimate the time (i.e., the number of iterations) necessary to reach 1 , starting from an arbitrary initial condition. Up to logarithmic corrections, this time, for a typical initial integer $n$, should simply be proportional to $\log [n]$. To properly test (but of course not prove) this conjecture, we need to sample over a very large ensemble of initial integers. Such a task, however, is beyond the scope of the present analysis.

Second, surely the Collatz dynamics is not an example of a natural process displaying genuine SOC. For instance, SOC usually (but see [25]) is defined in a spatial (geometric) medium, which of course is not the case for a plain sequence of integers. Nonetheless, we will point out some parallels between our purely mathematical rules and certain physical mechanisms generating SOC [17-19]. Commonly, SOC models alternate between slow input and fast dissipation bursts of energy. For the Collatz map, we could suppose $n_{j}$ to be some extensive quantity, proportional to energy. In this hypothetical interpretation, for $n$ large the gradual increase of energy might correspond to instances where $n \rightarrow n^{\prime} \approx \frac{3}{2} n$, whereas the fast release results from the avalanches $n \rightarrow n^{\prime}=n / 2^{m}$. Also, regardless of the starting condition [for the Collatz a typical very large $n_{0}$ (see Appendix A)], standard physical systems selforganize to the critical behavior after some time. Similarly, if for a particular integer the Collatz map takes it very far down, then the correspondingly lower density (of multiples of 2) makes the next avalanche likely to be smaller than otherwise expected. Thus, a typical hailstone sequence has a built-in negative feedback, e.g., the sandpile model. Hence, although the Collatz map cannot have SOC in the usual sense, it bears these striking (and unexpected) similarities.

Third, as in our model, scale-free behavior does not emerge only in systems tending to a stationary state $[25,48]$ or necessarily presenting randomness $[23,51]$. However, it would be 
worth asking how and why the positive integers yield such remarkable properties under such a simple linear map [52].

Fourth, an important signature in complex systems $[2,4,5,22]$ is long-range power-law correlations. Assuming the Collatz conjecture is true (but even if it is not, a very recent result [40] implies that infinite many $n_{0}$ satisfy the hypothesis), among all the infinite possible hailstone sequences, one should find orbits that contain a stretch of $M$ numbers with such a type of correlation, moreover with $M$ arbitrarily large. In fact, by means of a constructive example, we demonstrate in Appendix $\mathrm{C}$ that highly correlated stretches in the Collatz sequence actually can emerge. Hence, conceivably certain Collatz orbits could mimic statistical features of important classes of natural processes [7,9]. In this way, a relevant question is the following: Given the interval $\left\{n_{0}-\Delta, n_{0}+\Delta\right\}$, what is the number $\mathcal{N}\left(n_{0}, \Delta\right)$ of such orbits? Of course, if $\mathcal{N}$ is very small, the scale-free dynamics would be only marginal for the Collatz map. Surely, this is an investigation worth pursuing in future studies.

We finally mention some implications of our analysis for the validity of the Collatz conjecture (see [40]). The fact that the orbits appear as 1D RWs with drift on a logarithmic scale (related to the importance of the logarithmic scale, refer to the surprising model in [26]) strongly suggests that the absorbing boundary (here $n_{T}=1$ ) will always be reached. We further recall that, as it is well known in one and two dimensions, even a usual RW consistently returns to the origin because the root mean square displacement only scales as the square root of the number of steps. A possible strategy to prove the Collatz conjecture is to show that for any $n_{0}$, a finite $S$ such that $f^{(S)}\left(n_{0}\right)<n_{0}$ always exists [53]. This is closely associated with the first-passage-time problem for RWs [54]. Also, it would be interesting to study hailstone sequences from the perspective of the Andersen theorem [54], linking RWs and renewal theory.

Concluding, as already pointed out, the possible ubiquitousness of scale-free behavior in nature has motivated its search in very simple (but rigorous) purely mathematical models [15,24-26]. The hope is to find possibly universal rules that can lead to complexity, even independently of finetunable details. If successful, this kind of approach might be very useful to describe and understand countless realistic phenomena.

\section{ACKNOWLEDGMENTS}

We are grateful for fruitful discussions with L. S. Schulman, R. A. Caetano, T. Macrì, and F. Bohn. We also acknowledge important remarks and suggestions made by the anonymous referees. We thank CNPq (Grants No. 304532/2019-3, No. 305062/2017-4, and No. 302051/2018$0)$, CAPES, and FACEPE for funding.

M.G.E.d.L. and D.M.G.d.S. contributed equally to this work.

\section{APPENDIX A: DECOMPOSITION OF A NATURAL NUMBER IN A SPECIAL (AND UNIQUE) FORM OF POWERS OF 2}

Consider a natural number $n_{0} \geqslant 1$. Now assume that $m_{1}=$ $0,1,2, \ldots$ is the largest non-negative natural number for which $n_{0} / 2^{m_{1}}$ is an integer. Thus, we can write

$$
n_{0}=2^{m_{1}}\left(n_{1}+1\right) \text {, }
$$

where $n_{1}$ is uniquely determined (given the above condition for $m_{1}$ ). Also, $n_{1}$ is zero if $n_{0}$ is an exact power of 2 (and then the decomposition ends) or a non-null even number otherwise. In the latter case, we can further decompose $n_{1}$ as

$$
n_{1}=2^{m_{2}}\left(n_{2}+1\right)
$$

for $m_{2}=1,2,3, \ldots$, the largest non-null integer for which $n_{1} / 2^{m_{2}}$ is an integer (recall that $m_{2} \geqslant 1$ because $n_{1}$ is even). Again, $n_{2}$ is unique and either zero (so the decomposition ends) or even. This process continues until we reach $n_{r}=0$ for some $r=1,2,3, \ldots$. We therefore finally get

$$
n_{0}=2^{m_{1}}\left(\left(2^{m_{2}}\left(\left(2^{m_{3}}\left(\left(2^{m_{4}}\left(\left(\cdots\left(2^{m_{r}}\right)\right)+1\right)\right)+1\right)\right)+1\right)\right)+1\right)=2^{m_{1}}+2^{m_{1}+m_{2}}+2^{m_{1}+m_{2}+m_{3}}+\cdots+2^{m_{1}+m_{2}+m_{3}+\cdots+m_{r}} .
$$

The above representation of the integer $n_{0} \geqslant 1$ in terms of powers of 2 can be defined as

$$
n_{0} \equiv\left(m_{1}, m_{2}, m_{3}, \ldots, m_{r-1}, m_{r}\right)
$$

for the exponents $m_{i}(i=1,2, \ldots, r)$ being determined in a very precise way from the above-described rules. Here it is worth recalling that only $m_{1}$ can be null; all the other $m_{i}(i \geqslant$ 2) are necessarily greater than zero. Furthermore, if $m_{1}=0$ $\left(m_{1}>0\right)$ then $n_{0}$ is odd (even).

In fact, the following is a direct algorithm to find the $m_{i}$ 's in Eq. (A4) for any $n_{0} \geqslant 1$.
(a) Set $M=n_{0}$.
(b) Set $s=1$.
(c) Start the loop.
(c 0$)$ The loop halting condition $M=0$.

(c 1) Successively divide $M$ by 2 until finding $m$ such that $m$ is the largest integer for which $M / 2^{m}$ is an integer. (c 2) Set $m_{s}=m$.

(c 3) Update $M \rightarrow M / 2^{m}-1$.

(c 4) Update $s \rightarrow s+1$.

(c 5) Return to (c 0).

The converse, namely, writing a natural number in the usual base-10 system from the Eq. (A4) representation, follows directly from Eq. (A3).

\section{Values of the $\tilde{f}(n)$ Collatz map in the above power of 2 representation}

The action of the Collatz map $\tilde{f}$ [Eq. (5)] (the analysis for $f$ [Eq. (1)] is very similar) on a natural number $n$ is simple to portray when $n$ is given in the representation of Eq. (A4). Indeed, for $\tilde{f}$ defined by

$$
\tilde{f}(n)= \begin{cases}n / 2, & n \text { even } \\ n+(n+1) / 2, & n \text { odd }\end{cases}
$$


it is not difficult to see from Eq. (A3) that for $n=$ $\left(m_{1}, m_{2}, m_{3}, \ldots, m_{r-2}, m_{r-1}, m_{r}\right)$, if $n$ is even (so that $m_{1} \geqslant$ 1) then

$$
\frac{n}{2}=\left(m_{1}-1, m_{2}, m_{3}, \ldots, m_{r-2}, m_{r-1}, m_{r}\right),
$$

whereas for $n$ odd (hence $m_{1}=0$ )

$$
\begin{aligned}
n+\frac{n+1}{2}= & \left(m_{1}, m_{2}, m_{3}, \ldots, m_{r-2}, m_{r-1}, m_{r}\right) \\
& \oplus\left(m_{1}, m_{2}-1, m_{3}, \ldots, m_{r-2}, m_{r-1}, m_{r}\right) .
\end{aligned}
$$

Here we should interpret $\oplus$ as the proper way to sum two natural numbers written in the present specific power of 2 representation. The first term on the right-hand side of Eq. (A7) is simply the representation of $n$ and the second is the representation of $(n+1) / 2$.

Presently, we are still working on a possible analytical method to obtain $m_{1}^{\prime}, m_{2}^{\prime}, m_{3}^{\prime}, \ldots$, where

$$
\begin{aligned}
\left(m_{1}^{\prime}, m_{2}^{\prime}, m_{3}^{\prime}, \ldots\right)= & \left(m_{1}, m_{2}, m_{3}, \ldots, m_{r-2}, m_{r-1}, m_{r}\right) \\
& \oplus\left(m_{1}, m_{2}-1, m_{3}, \ldots, m_{r-2}, m_{r-1}, m_{r}\right) .
\end{aligned}
$$

Nevertheless, for all the numerical calculations we can write the right-hand side of Eq. (A8) as

$$
\begin{gathered}
\left(2^{m_{1}}+2^{m_{1}+m_{2}}+\cdots+2^{m_{1}+\cdots+m_{r}}\right)+\left(2^{m_{1}}+2^{m_{1}+m_{2}-1}\right. \\
\left.+2^{m_{1}+m_{2}-1+m_{3}}+\cdots+2^{m_{1}+m_{2}-1+m_{3}+\cdots+m_{r}}\right)
\end{gathered}
$$

and then apply the algorithm discussed above (with few extra, but simple, steps) to compute the exponent set $m_{1}^{\prime}, m_{2}^{\prime}, m_{3}^{\prime}, \ldots$.

\section{The $n_{0}$ 's used to generate typical orbits}

As mentioned in the main text, it is important to choose appropriate very large $n_{0}$ 's to guarantee the presence of scale- free behavior in the corresponding generated orbits $\mathcal{O}\left(n_{0}\right)$, as well as to be able to perform reliable statistical analysis. Appropriate here does not mean very special particular instances. On the contrary, it refers to $n_{0}$ 's giving rise to "typical" hailstone sequences, i.e., with fairly heterogeneous patterns of up and down fluctuations. Moreover they usually need to pass through intermediate $n_{j}$ 's that can be much larger than, e.g., one googol $\left(10^{100}\right)$.

The six examples given in this work result from typical $n_{0}$ 's. In fact, by picking at will a certain huge $n_{0}$, in the great majority of the cases the resulting $\mathcal{O}\left(n_{0}\right)$ 's will be appropriate to our study. However, obviously, one may choose an $n_{0}$ leading to too structured orbits, e.g., of very short length or with a very repetitive trivial succession of ascents and descents in their hailstone sequence. These would be nontypical situations.

However, by numerically playing with the orbits we have observed some potential (heuristic) criteria for good $n_{0}$ candidates. Although we still do not have a solid theoretical explanation for these eventual criteria, it seems that if the exponent set $\left\{m_{i}\right\}$ of $n_{0}$ consists of repeated short sequences of primes, such as $2,3,5$ or $5,7,11$, or even the simple case of 1,2, 3 (of course here with 1 not prime) and from time to time we have a stretch of $m_{i}$ 's all equal to 2's (also prime), then the resulting $\mathcal{O}\left(n_{0}\right)$ will be "typical"; here we should observe that the very simple example of SOC in [25] is also based on the generation of prime numbers. However, this type of prime-based pattern is not unique to giving rise to a power law for the hailstone sequences. In certain instances, unexpectedly, particular numbers such as 77 should appear repeatedly in the $\left\{m_{i}\right\}$ set, which is exactly the example for $n_{0}^{(4)}$ below.

Finally, we comment that we do believe that the behavior of the $\left\{m_{i}\right\}$ 's themselves (under the action of the Collatz map) might constitute an important hint to finally settle the Collatz conjecture.

The set of $\left\{m_{i}\right\}$ 's of the six $n_{0}$ used in our study is the following (recall that all are odd numbers, hence always $m_{1}=0$ ):

$$
\begin{aligned}
n_{0}^{(1)}= & (0,1,2,3,1,2,3,1,2,3,1,2,3,1,2,3,1,2,3,1,2,3,1,2,3,1,2,3,1,2,3), \\
n_{2}^{(0)}= & (0,2,3,5,2,3,5,2,3,5,2,3,5,2,3,5,2,3,5,2,3,5,2,3,5,2,3,5,2,3,5,2,3,5, \\
& 2,3,5,2,3,5,2,3,5,2,3,5,2,3,5,2,3,5,2,3,5,2,3,5,2,3,5,2,3,5,2,3,5, \\
& 2,3,5,2,3,5,2,3,5,2,3,5,2,3,5,2,3,5,2,3,5,2,3,5,2,3,5,2,3,5,2,3,5, \\
& 2,3,5,2,3,5) \\
n_{3}^{(0)}= & (0,2,3,5,2,3,5,2,3,5,2,3,5,2,3,5,2,3,5,2,3,5,2,3,5,2,3,5,2,3,5,2,3,5, \\
& 2,3,5,2,3,5,2,3,5,2,3,5,2,3,5,2,3,5,2,3,5,2,3,5,2,3,5,2,3,5,2,3,5, \\
& 2,3,5,2,3,5,2,3,5,2,3,5,2,3,5,2,3,5,2,3,5,2,3,5,2,3,5,2,3,5,2,3,5, \\
& 2,3,5,2,3,5,2,3,5,2,3,5,2,3,5,2,3,5,2,3,5,2,3,5,2,3,5,2,3,5,2,3,5, \\
& 2,3,5,2,3,5,2,3,5,2,3,5,2,3,5,2,3,5,2,3,5,2,3,5), \\
n_{0}^{(4)}= & (0,3,5,7,77,3,5,7,77,3,5,7,77,3,5,7,77,3,5,7,77,3,5,7,77), \\
n_{0}^{(5)}= & (0,5,7,11,5,7,11,5,7,11,5,7,11,5,7,11,5,7,11,5,7,11,5,7,11,5,7,11,5,7,11, \\
& 5,7,11,5,7,11,5,7,11,5,7,11,5,7,11,5,7,11,5,7,11,5,7,11,5,7,11,5,7,11, \\
& 5,7,11,5,7,11,5,7,11,5,7,11,5,7,11,5,7,11,5,7,11,5,7,11,5,7,11,5,7,11,
\end{aligned}
$$


$5,7,11,5,7,11,5,7,11,5,7,11,2,2,2,2,2,2,2,2,2,2,2,5,7,11,5,7,11,5,7,11$, $5,7,11,5,7,11,5,7,11,5,7,11,5,7,11,5,7,11,5,7,11,5,7,11,5,7,11,5,7,11$, $5,7,11,5,7,11,5,7,11,5,7,11,5,7,11,5,7,11,5,7,11,5,7,11,5,7,11,5,7,11$, $5,7,11,5,7,11,5,7,11,5,7,11,5,7,11,5,7,11,5,7,11,5,7,11,5,7,11,5,7,11$, $5,7,11,5,7,11,5,7,11,5,7,11,5,7,11,5,7,11,5,7,11,5,7,11,5,7,11,5,7,11$ $5,7,11,5,7,11,5,7,11,5,7,11,5,7,11,5,7,11,5,7,11,5,7,11,5,7,11,5,7,11$, $5,7,11,5,7,11,5,7,11,5,7,11,5,7,11,5,7,11,5,7,11,5,7,11,5,7,11,5,7,11$, $5,7,11,5,7,11,5,7,11,5,7,11,5,7,11,2,2,2,2,2,2,2,2,2,2,2,5,7,11,5,7,11$, $5,7,11,5,7,11,5,7,11,5,7,11,5,7,11,5,7,11,5,7,11,5,7,11,5,7,11,5,7,11$, $5,7,11,5,7,11,5,7,11,5,7,11,5,7,11,5,7,11,5,7,11,5,7,11,5,7,11,5,7,11$, $5,7,11,5,7,11,5,7,11,5,7,11,5,7,11,5,7,11,5,7,11,5,7,11,5,7,11,5,7,11$, $5,7,11,5,7,11,5,7,11,5,7,11,5,7,11,5,7,11,5,7,11,5,7,11,5,7,11,5,7,11$, $5,7,11,5,7,11,5,7,11,5,7,11,5,7,11,5,7,11,5,7,11,5,7,11,5,7,11,5,7,11$, $5,7,11,5,7,11,5,7,11,5,7,11,5,7,11,5,7,11,5,7,11,5,7,11,5,7,11,5,7,11$, $5,7,11,5,7,11,5,7,11,5,7,11,5,7,11,5,7,11,2,2,2,2,2,2,2,2,2,2,2,5,7,11$, $5,7,11,5,7,11,5,7,11,5,7,11,5,7,11,5,7,11,5,7,11,5,7,11,5,7,11,5,7,11$, $5,7,11,5,7,11,5,7,11,5,7,11,5,7,11,5,7,11,5,7,11,5,7,11,5,7,11,5,7,11$, $5,7,11,5,7,11,5,7,11,5,7,11,5,7,11,5,7,11,5,7,11,5,7,11,5,7,11,5,7,11$, $5,7,11,5,7,11,5,7,11,5,7,11,5,7,11,5,7,11,5,7,11,5,7,11,5,7,11,5,7,11$, $5,7,11,5,7,11,5,7,11,5,7,11,5,7,11,5,7,11,5,7,11,5,7,11,5,7,11,5,7,11$, $5,7,11,5,7,11,5,7,11,5,7,11,5,7,11,5,7,11,5,7,11,5,7,11,5,7,11,5,7,11$, $5,7,11,5,7,11,5,7,11,5,7,11,5,7,11,5,7,11,5,7,11,2,2,2,2,2,2,2,2,2,2,2$, $5,7,11,5,7,11,5,7,11,5,7,11,5,7,11,5,7,11,5,7,11,5,7,11,5,7,11,5,7,11$, $5,7,11,5,7,11,5,7,11,5,7,11,5,7,11,5,7,11,5,7,11,5,7,11,5,7,11,5,7,11$, $5,7,11,5,7,11,5,7,11,5,7,11,5,7,11,5,7,11,5,7,11,5,7,11,5,7,11,5,7,11$, $5,7,11,5,7,11,5,7,11,5,7,11,2,2,2,2,2,2)$,

$n_{0}^{(6)}=(0,5,7,11,5,7,11,5,7,11,5,7,11,5,7,11,5,7,11,5,7,11,5,7,11,5,7,11,5,7,11$, $5,7,11,5,7,11,5,7,11,5,7,11,5,7,11,5,7,11,5,7,11,5,7,11,5,7,11,5,7,11$, $5,7,11,5,7,11,5,7,11,5,7,11,5,7,11,5,7,11,5,7,11,5,7,11,5,7,11,5,7,11$, $5,7,11,5,7,11,5,7,11,5,7,11,2,2,2,2,2,2,2,2,2,2,2,5,7,11,5,7,11,5,7,11$, $5,7,11,5,7,11,5,7,11,5,7,11,5,7,11,5,7,11,5,7,11,5,7,11,5,7,11,5,7,11$, $5,7,11,5,7,11,5,7,11,5,7,11,5,7,11,5,7,11,5,7,11,5,7,11,5,7,11,5,7,11$, $5,7,11,5,7,11,5,7,11,5,7,11,5,7,11,5,7,11,5,7,11,5,7,11,5,7,11,5,7,11$, $5,7,11,5,7,11,5,7,11,5,7,11,5,7,11,5,7,11,5,7,11,5,7,11,5,7,11,5,7,11$, $5,7,11,5,7,11,5,7,11,5,7,11,5,7,11,5,7,11,5,7,11,5,7,11,5,7,11,5,7,11$, $5,7,11,5,7,11,5,7,11,5,7,11,5,7,11,5,7,11,5,7,11,5,7,11,5,7,11,5,7,11$, $5,7,11,5,7,11,5,7,11,5,7,11,5,7,11,2,2,2,2,2,2,2,2,2,2,2,5,7,11,5,7,11$, $5,7,11,5,7,11,5,7,11,5,7,11,5,7,11,5,7,11,5,7,11,5,7,11,5,7,11,5,7,11$, $5,7,11,5,7,11,5,7,11,5,7,11,5,7,11,5,7,11,5,7,11,5,7,11,5,7,11,5,7,11$, $5,7,11,5,7,11,5,7,11,5,7,11,5,7,11,5,7,11,5,7,11,5,7,11,5,7,11,5,7,11$, $5,7,11,5,7,11,5,7,11,5,7,11,5,7,11,5,7,11,5,7,11,5,7,11,5,7,11,5,7,11$, $5,7,11,5,7,11,5,7,11,5,7,11,5,7,11,5,7,11,5,7,11,5,7,11,5,7,11,5,7,11$, 
$5,7,11,5,7,11,5,7,11,5,7,11,5,7,11,5,7,11,5,7,11,5,7,11,5,7,11,5,7,11$, $5,7,11,5,7,11,5,7,11,5,7,11,5,7,11,5,7,11,2,2,2,2,2,2,2,2,2,2,2,5,7,11$, $5,7,11,5,7,11,5,7,11,5,7,11,5,7,11,5,7,11,5,7,11,5,7,11,5,7,11,5,7,11$, $5,7,11,5,7,11,5,7,11,5,7,11,5,7,11,5,7,11,5,7,11,5,7,11,5,7,11,5,7,11$, $5,7,11,5,7,11,5,7,11,5,7,11,5,7,11,5,7,11,5,7,11,5,7,11,5,7,11,5,7,11$, $5,7,11,5,7,11,5,7,11,5,7,11,5,7,11,5,7,11,5,7,11,5,7,11,5,7,11,5,7,11$, $5,7,11,5,7,11,5,7,11,5,7,11,5,7,11,5,7,11,5,7,11,5,7,11,5,7,11,5,7,11$, $5,7,11,5,7,11,5,7,11,5,7,11,5,7,11,5,7,11,5,7,11,5,7,11,5,7,11,5,7,11$, $5,7,11,5,7,11,5,7,11,5,7,11,5,7,11,5,7,11,5,7,11,2,2,2,2,2,2,2,2,2,2,2$, $5,7,11,5,7,11,5,7,11,5,7,11,5,7,11,5,7,11,5,7,11,5,7,11,5,7,11,5,7,11$, $5,7,11,5,7,11,5,7,11,5,7,11,5,7,11,5,7,11,5,7,11,5,7,11,5,7,11,5,7,11$, $5,7,11,5,7,11,5,7,11,5,7,11,5,7,11,5,7,11,5,7,11,5,7,11,5,7,11,5,7,11$, $5,7,11,5,7,11,5,7,11,5,7,11,2,2,2,2,2,2,5,7,11,5,7,11,5,7,11,5,7,11$, $5,7,11,5,7,11,5,7,11,5,7,11,5,7,11,5,7,11,5,7,11,5,7,11,5,7,11,5,7,11$, $5,7,11,5,7,11,5,7,11,5,7,11,5,7,11,5,7,11,5,7,11,5,7,11,5,7,11,5,7,11$, $5,7,11,5,7,11,5,7,11,5,7,11,5,7,11,5,7,11,5,7,11,5,7,11,5,7,11,5,7,11$, $2,2,2,2,2,2,2,2,2,2,2,5,7,11,5,7,11,5,7,11,5,7,11,5,7,11,5,7,11,5,7,11$, $5,7,11,5,7,11,5,7,11,5,7,11,5,7,11,5,7,11,5,7,11,5,7,11,5,7,11,5,7,11$, $5,7,11,5,7,11,5,7,11,5,7,11,5,7,11,5,7,11,5,7,11,5,7,11,5,7,11,5,7,11$, $5,7,11,5,7,11,5,7,11,5,7,11,5,7,11,5,7,11,5,7,11,5,7,11,5,7,11,5,7,11$, $5,7,11,5,7,11,5,7,11,5,7,11,5,7,11,5,7,11,5,7,11,5,7,11,5,7,11,5,7,11$, $5,7,11,5,7,11,5,7,11,5,7,11,5,7,11,5,7,11,5,7,11,5,7,11,5,7,11,5,7,11$, $5,7,11,5,7,11,5,7,11,5,7,11,5,7,11,5,7,11,5,7,11,5,7,11,5,7,11,5,7,11$, $5,7,11,2,2,2,2,2,2,2,2,2,2,2,5,7,11,5,7,11,5,7,11,5,7,11,5,7,11,5,7,11$, $5,7,11,5,7,11,5,7,11,5,7,11,5,7,11,5,7,11,5,7,11,5,7,11,5,7,11,5,7,11$, $5,7,11,5,7,11,5,7,11,5,7,11,5,7,11,5,7,11,5,7,11,5,7,11,5,7,11,5,7,11$, $5,7,11,5,7,11,5,7,11,5,7,11,5,7,11,5,7,11,5,7,11,5,7,11,5,7,11,5,7,11$, $5,7,11,5,7,11,5,7,11,5,7,11,5,7,11,5,7,11,5,7,11,5,7,11,5,7,11,5,7,11$, $5,7,11,5,7,11,5,7,11,5,7,11,5,7,11,5,7,11,5,7,11,5,7,11,5,7,11,5,7,11$, $5,7,11,5,7,11,5,7,11,5,7,11,5,7,11,5,7,11,5,7,11,5,7,11,5,7,11,5,7,11$, $5,7,11,5,7,11,2,2,2,2,2,2,2,2,2,2,2,5,7,11,5,7,11,5,7,11,5,7,11,5,7,11$, $5,7,11,5,7,11,5,7,11,5,7,11,5,7,11,5,7,11,5,7,11,5,7,11,5,7,11,5,7,11$, $5,7,11,5,7,11,5,7,11,5,7,11,5,7,11,5,7,11,5,7,11,5,7,11,5,7,11,5,7,11$, $5,7,11,5,7,11,5,7,11,5,7,11,5,7,11,5,7,11,5,7,11,5,7,11,5,7,11,5,7,11$, $5,7,11,5,7,11,5,7,11,5,7,11,5,7,11,5,7,11,5,7,11,5,7,11,5,7,11,5,7,11$, $5,7,11,5,7,11,5,7,11,5,7,11,5,7,11,5,7,11,5,7,11,5,7,11,5,7,11,5,7,11$, $5,7,11,5,7,11,5,7,11,5,7,11,5,7,11,5,7,11,5,7,11,5,7,11,5,7,11,5,7,11$, $5,7,11,5,7,11,5,7,11,2,2,2,2,2,2,2,2,2,2,2,5,7,11,5,7,11,5,7,11,5,7,11$, $5,7,11,5,7,11,5,7,11,5,7,11,5,7,11,5,7,11,5,7,11,5,7,11,5,7,11,5,7,11$, $5,7,11,5,7,11,5,7,11,5,7,11,5,7,11,5,7,11,5,7,11,5,7,11,5,7,11,5,7,11$, $5,7,11,5,7,11,5,7,11,5,7,11,5,7,11,5,7,11,5,7,11,5,7,11,5,7,11,5,7,11$, $2,2,2,2,2,2)$. 


\section{APPENDIX B: ANALYTICAL ESTIMATION OF $\gamma$}

In Sec. III we numerically calculated a mean value for $\gamma$ using 50 randomly generated orbits. Here we present an analytic estimation for this exponent.

Observe the following (a known argument in favor of the Collatz conjecture [33]). Whenever the $j$ th Collatz hailstone term $n_{j}$ is even, then (e) the subsequently is $n_{j+1}=f\left(n_{j}\right)=$ $n_{j} / 2$, which can be either even or odd. However, if $n_{j}$ is odd, then (o) $n_{j+1}=f\left(n_{j}\right)=3 n_{j}+1$ is necessarily even, so $n_{j+2}=f\left(n_{j+1}\right)=\left(3 n_{j}+1\right) / 2$, which again can be even or odd. Since evens and odds are equally distributed in $\mathbb{N}$, process (e) takes one step, process (o) takes two steps, and for (o) and $n_{j}$ large, $n_{j+2} \sim \frac{3}{2} n_{j}$, we find that on average after $s=3$ steps an integer $n_{j}$ is reduced to $\frac{1}{2} \times \frac{3}{2} n_{j}=\frac{3}{4} n_{j}$.

Suppose now that starting with $n_{0}$, we have a steady decrease of $n_{0}$ by a factor $\frac{3}{4}$ in each step or

$$
n_{0} \rightarrow\left(\frac{3}{4}\right) n_{0} \rightarrow\left(\frac{3}{4}\right)^{2} n_{0} \rightarrow \cdots \rightarrow\left(\frac{3}{4}\right)^{\tilde{T}} n_{0} \approx 1
$$

The total number of terms (or steps) $\tilde{T}$ in the above monotonic sequence is associated with the initial number $n_{0}$ through

$$
\log \left[\left(\frac{3}{4}\right)^{\tilde{T}} n_{0}\right]=\log [1] \text { or } \log \left[n_{0}\right]=(3 \tilde{T}) \frac{\log \left[\frac{4}{3}\right]}{3}
$$

However, from the previous considerations, the main number of steps of $\left\{f^{(1)}\left(n_{0}\right), \ldots, f^{(T)}\left(n_{0}\right)=1\right\}$ is $T=s \tilde{T}=3 \tilde{T}$. Thus, given the definition of $\gamma$ in Sec. III and Eq. (B1),

$$
\gamma=\frac{1}{3} \log \left[\frac{4}{3}\right] \approx 0.041646
$$

\section{APPENDIX C: EXAMPLE OF THE EXISTENCE OF A LONG CORRELATED STRETCH IN A COLLATZ HAILSTONE SEQUENCE}

Here we give an explicit example of a long stretch in a Collatz sequence that can lead to distributions similar to those of time series associated with strongly correlated systems. We emphasize that, on the one hand, our example does not exhaust all the possibilities of correlations in the Collatz hailstones. On the other hand, it also does not prove that such correlations commonly arise in $\left\{f^{(S)}\left(n_{0}\right)\right\}$ if $n_{0}$ is arbitrarily large. With the present analysis our aim is limited to giving concrete support to our speculations in Sec. IV (see the fourth point in the Conclusion), namely, the Collatz map might be a surprising generator of long-range power-law correlated series, thus somehow emulating the dynamics of complex systems.

We define $\Gamma_{i}=\sum_{j=0}^{j=i}\left(\Delta_{j}+k_{j}\right)(i=0,1,2, \ldots)$ for $\Delta_{0}=$ $r_{0}=0$ (so $\Gamma_{0}=0$ ) and $\Delta, \Gamma$, and $k$ all non-negative integers. In the following a "step" means a single application of the Collatz map $f$. Suppose we start with a very large (and special, discussed below) number $n_{0}=n_{\Gamma_{0}}$, yielding the following hailstone pattern (for odd $\mathcal{F}_{i}$ ):

$$
\begin{aligned}
& n_{\Gamma_{0}} \stackrel{\Delta_{1} \text { steps }}{\longrightarrow} n_{\Gamma_{0}+\Delta_{1}}=2^{k_{1}} \mathcal{F}_{1} \stackrel{k_{1} \text { steps }}{\longrightarrow} n_{\Gamma_{1}}=\mathcal{F}_{1}, \\
& n_{\Gamma_{1}} \stackrel{\Delta_{2} \text { steps }}{\longrightarrow} n_{\Gamma_{1}+\Delta_{2}}=2^{k_{2}} \mathcal{F}_{2} \stackrel{k_{2} \text { steps }}{\longrightarrow} n_{\Gamma_{2}}=\mathcal{F}_{2}, \\
& n_{\Gamma_{2}} \stackrel{\Delta_{3} \text { steps }}{\longrightarrow} n_{\Gamma_{2}+\Delta_{3}}=2^{k_{3}} \mathcal{F}_{3} \stackrel{k_{3} \text { steps }}{\longrightarrow} n_{\Gamma_{3}}=\mathcal{F}_{3}, \\
& n_{\Gamma_{3}} \stackrel{\Delta_{4} \text { steps }}{\longrightarrow} n_{\Gamma_{3}+\Delta_{4}}=2^{k_{4}} \mathcal{F}_{4} \stackrel{k_{4} \text { steps }}{\longrightarrow} n_{\Gamma_{4}}=\mathcal{F}_{4},
\end{aligned}
$$

Eventually the above repetitive structure could terminate after the $\Gamma_{I}$ th step. Nonetheless, for $I$ big enough, Eq. (C1) is our sought long stretch of the Collatz sequence.

For the $k_{i}$ 's obeying some particular distribution, say, a power law, the successive avalanches in the Collatz sequence, as defined in the present work, would display scale-invariance behavior. Likewise, the $\Delta_{i}$ 's (the duration between the powerlaw decays) could also present some specific distribution. The key point is that all this may take place, but only depending on the fine-tuning characteristics of the $\mathcal{F}_{i}$ 's. We are not aware of general conditions resulting in such proper sets $\left\{\mathcal{F}_{i}\right\}$. However, next we propose a particular instance where Eq. (C1) with strong correlations can emerge.

Let us make the workable assumptions that for all $i$, (i) $k_{i}$ is even, (ii) $\mathcal{F}_{i}=6 F_{i}^{(0)}+1$ is an odd integer, and (iii) $F_{i}^{(0)}=$ $4^{j_{i}} E_{i}$ for $j_{i}=1,2, \ldots$ and $E_{i}$ odd. Of course, we must verify that these suppositions are self-consistent and conform with Eq. (C1). Assuming this is the case, for an arbitrary $i$,

$$
6 F_{i}^{(0)}+1 \stackrel{3 \text { steps }}{\longrightarrow} \frac{9 F_{i}^{(0)}}{2}+1=6 F_{i}^{(1)}+1, \quad F_{i}^{(1)}=\frac{3}{4} F_{i}^{(0)} .
$$

If $F_{i}^{(1)}$ is still even, we once more get

$$
6 F_{i}^{(1)}+1 \stackrel{3 \text { steps }}{\longrightarrow} \frac{9 F_{i}^{(1)}}{2}+1=6 F_{i}^{(2)}+1, \quad F_{i}^{(2)}=\frac{3}{4} F_{i}^{(1)},
$$

and so forth. This recursive calculation repeats until

$$
F_{i}^{\left(j_{i}\right)}=\left(\frac{3}{4}\right)^{j_{i}} F_{i}^{(0)}
$$

is ultimately odd because of assumption (iii) above.

Upon reaching $6 F_{i}^{\left(j_{i}\right)}+1\left(\right.$ from $\left.\mathcal{F}_{i}\right)$, we have

$$
6 F_{i}^{\left(j_{i}\right)}+1 \stackrel{3 \text { steps }}{\longrightarrow} 3^{3} F_{i}^{\left(j_{i}\right)}+7=\frac{3^{j_{i}+3}}{4^{j_{i}}} F_{i}^{(0)}+7
$$

for the latter number being even. Finally, we suppose the assumption (iv),

$$
\frac{3^{j_{i}+3}}{4^{j_{i}}} F_{i}^{(0)}+7=2^{k_{i+1}} \mathcal{F}_{i+1}=2^{k_{i+1}}\left(6 F_{i+1}^{(0)}+1\right),
$$

or [considering (iii) for $F_{i}^{(0)}$ ]

$$
2^{k_{i+1}+1} F_{i+1}^{(0)}=3^{j_{i}+2} E_{i}-\frac{2^{k_{i+1}}-1}{3}+2 .
$$

From (i) $k_{i+1}$ is even; then it is an elementary result that $2^{k_{i+1}}-1$ is an odd number divisible by 3 . Since $3^{j_{i}+2} E_{i}$ is also odd, the right-hand side of Eq. (C4) is even. Thus, it is always possible to choose a large $E_{i}$ (actually, there are infinite 
solutions ${ }^{1}$ ) such that the right-hand side of Eq. (C4) is equal to $2^{v} E_{i+1}$, with $v$ a big odd integer. Thus, consistently, we end up with $F_{i+1}^{(0)}=4^{j_{i+1}} E_{i+1}$ for $2 j_{i+1}=v-k_{i+1}-1$. These are just the previously considered assumptions (i)-(iii), which once valid for $i$ are also valid for $i+1$. Furthermore, note that $\Delta_{i+1}$ in Eq. (C1) is entirely related to the value of $j_{i}$ in Eq. (C2).

In summary, the conditions (i)-(iii) are recursively consistent with Eq. (C1) for any $i=1,2, \ldots, I$ (with $I$ arbitrary). Similarly relevant, the assumption (iv) leads to a functional relation between the parameters of Eq. (C1) in the form [cf. Eq. (C3)]

$$
G\left(k_{i+1}, j_{i+1}, E_{i+1} ; j_{i}, E_{i}\right)=0 .
$$

\footnotetext{
${ }^{1}$ This statement is essentially equivalent to show that, regardless of the integers $u$ and $B$ (with $B$ moreover odd), the expression $3^{u} A-B$ can always be written as $2^{v} C$ ( $v$ and $C$ odd integers) by properly choosing an odd integer $A$ (actually, there are infinite many $A$ 's satisfying the proposition, each yielding a particular $v$ and $C$ ). The proof can be done recursively, but we omit it here. However, we mention that this result is easy to check by playing with simple numerical simulations.
}

We notice that although the $E_{i}$ 's are constrained by Eq. (C5), there is great freedom for their possible values (especially if they are allowed to be very large). Hence, mathematically, one can have $\left\{k_{i}\right\}$ (in effect $\left\{k_{i} / 2\right\}$ ) following, say, a power-law distribution and still verify Eq. (C5) through proper $\left\{E_{i}\right\}$ 's (this is further reinforced by the fact that the $j_{i}$ 's are partially free due to the $v$ dependence above). One finally guarantees that Eq. (C1) occurs in a Collatz hailstone sequence, for instance, by starting with $n_{0}=\mathcal{F}_{1}$.

The aforementioned attainable numerical structure for long intervals of $\left\{f^{(S)}\left(n_{0}\right)\right\}$ illustrates that stretches of the Collatz sequence can indeed exhibit scale-free correlations. Certainly the present constructive demonstration is not practical from a numerical point of view. However, the central point is that they do exist. Thus, conceivably other numerical arrangements could also give rise to similar features. This naturally introduces the question of how frequently sequences with scale-free properties, somehow akin to complex systems, should emerge in the Collatz dynamics.
[1] W. Weaver, Science and complexity, Am. Sci. 36, 536 (1948).

[2] Y. B.- Yam, in Knowledge Management, Organizational Intelligence and Learning, and Complexity, edited by L. D. Kiel (Unesco-EOLSS, New York, 2007), Vol. 1, pp. 43-95.

[3] J. Ladyman, J. Lambert, and K. Wiesner, What is a complex system? Eur. J. Philos. Sci. 3, 33 (2013).

[4] R. Badii and A. Politi, Complexity: Hierarchical Structures and Scaling in Physics, 2nd ed. (Cambridge University Press, Cambridge, 1999).

[5] Complex dynamics of life at different scales: From genomic to global environmental issues, edited by C. Anteneodo and M. G. E. da Luz, special issue of Philos. Trans. R. Soc. A $\mathbf{3 6 8}$, 5559 (2010).

[6] Y. I. Wolf, M. I. Katsnelson, and E. V. Koonin, Physical foundations of biological complexity, Proc. Natl. Acad. Sci. U.S.A. 115, E8678 (2018).

[7] M. Sales-Pardo, R. Guimerá, A. A. Moreira, and L. A. N. Amaral, Extracting the hierarchical organization of complex systems, Proc. Natl. Acad. Sci. U.S.A. 104, 15224 (2007).

[8] L. P. Kadanoff, Statistical Physics: Statics, Dynamics and Renormalization (World Scientific, Singapore, 2000).

[9] K. Christensen and N. R. Moloney, Complexity and Criticality (Imperial College Press, London, 2005).

[10] P. Bak, C. Tang, and K. Wiesenfeld, Self-Organized Criticality: An Explanation of the $1 / f$ Noise, Phys. Rev. Lett. 59, 381 (1987).

[11] P. Bak, C. Tang, and K. Wiesenfeld, Self-organized criticality, Phys. Rev. A 38, 364 (1988).

[12] J. S. Shiner, Self-organized criticality: Self-organized complexity? The disorder and "simple complexities" of power law distributions, Open Syst. Inf. Dyn. 7, 131 (2000).
[13] N. W. Watkins, G. Pruessner, S. C. Chapman, N. B. Crosby, and H. J. Jensen, 25 years of self-organized criticality: Concepts and controversies, Space Sci. Rev. 198, 3 (2016).

[14] C. Letellier, I. Leyva, and I. Sendiña-Nadal, Dynamical complexity measure to distinguish organized from disorganized dynamics, Phys. Rev. E 101, 022204 (2020).

[15] V. A. Golyk, Self-organized criticality, http://www.mit.edu/ 8. 334/grades/projects/projects12/V.\%20A.\%20Golyk.pdf (MIT, Cambridge, 2012).

[16] P. Bak, How Nature Works: The Science of Self-Organized Criticality (Springer, New York, 2013).

[17] H. Flyvbjerg, Simplest Possible Self-Organized Critical System, Phys. Rev. Lett. 76, 940 (1996).

[18] H. Flyvbjerg, Self-organized critical pinball machine, Physica A 340, 552 (2004).

[19] R. Dickman, M. A. Muñoz, A. Vespignani, and S. Zapperi, Paths to self-organized criticality, Braz. J. Phys. 30, 27 (2000).

[20] J. Feder, The evidence for self-organized criticality in sandiple dynamics, Fractals 03, 431 (1995).

[21] M. S. Vieira, Breakdown of self-organized criticality in sandpiles, Phys. Rev. E 66, 051306 (2002).

[22] D. Sornette, Critical Phenomena in Natural Science (Springer, Berlin, 2000).

[23] M. S. Vieira, Simple deterministic self-organized critical system, Phys. Rev. E 61, R6056 (2000).

[24] R. Cerf and M. Gorny, A Curie-Weiss model of self-organized criticality, Ann. Probab. 44, 444 (2016).

[25] B. Luque, O. Miramontes, and L. Lacasa, Number Theoretic Example of Scale-Free Topology Inducing Self-Organized Criticality, Phys. Rev. Lett. 101, 158702 (2008).

[26] N. Kalinin, A. Guzmán-Sáenz, Y. Prieto, M. Shkolnikov, V. Kalinina, and E. Lupercio, Self-organized criticality and pattern 
emergence through the lens of tropical geometry, Proc. Natl. Acad. Sci. U.S.A. 115, E8135 (2018).

[27] G. Caldarelli, A. Capocci, P. De Los Rios, and M. A. Muñoz, Scale-Free Networks from Varying Vertex Intrinsic Fitness, Phys. Rev. Lett. 89, 258702 (2002).

[28] Y. Zou, J. Heitzig, R. V. Donner, J. F. Donges, J. D. Farmer, R. Meucci, S. Euzzor, N. Marwan, and J. Kurths, Power-laws in recurrence networks from dynamical systems, Europhys. Lett. 98, 48001 (2012).

[29] D. L. Hu, W. Chen, and H. G. Sun, Power-law stability of Hausdorff derivative nonlinear dynamics systems, Int. J. Syst. Sci. 51, 601 (2020)

[30] K. Klemm and V. M. Eguíluz, Growing scale-free networks with small-world behavior, Phys. Rev. E 65, 057102 (2002).

[31] H. Zhou and R. Lipowsky, Dynamic pattern evolution on scale-free networks, Proc. Natl. Acad. Sci. U.S.A. 102, 10052 (2005).

[32] A. D. Broido and A. Clauset, Scale-free networks are rare, Nat. Commun. 10, 1017 (2019).

[33] The Ultimate Challenge: The $3 x+1$ Problem, edited by J. C. Lagarias (American Mathematical Society, Providence, 2011).

[34] R. K. Guy, Unsolved Problems in Number Theory (Springer, New York, 2004).

[35] J. C. Lagarias, The $3 x+1$ problem and its generalizations, Am. Math. Mon. 92, 3 (1985).

[36] M. Orús-Lacort, Analyzing Collatz conjecture with mathematical complete induction method, 2018 (unpublished).

[37] M. Chamberland, Averaging structure in the $3 x+1$ problem, https://pdfs.semanticscholar.org/d18d/ 00fbc624a9c0b1e6b958cf593e05122f11fd.pdf (2014).

[38] T. Oliveira e Silva, Computational verification of the $3 x+1 \quad$ conjecture, http://sweet.ua.pt/tos/3x_plus_1.html (2015).

[39] W. Ren, S. Li, R. Xiao, and W. Bi, Proceedings of the IEEE UIC 2018 (IEEE, Piscataway, 2018), pp. 411-416.

[40] T. Tao, Almost all orbits of the Collatz map attain almost bounded values, arXiv:1909.03562.
[41] T. Tao, The Collatz conjecture, Littlewood-Offord theory, and powers of 2 and 3, https://terrytao.wordpress.com/2011/08/25/ the-collatz-conjecture-littlewood-offord-theory-and-powersof-2-and-3/ (2011).

[42] M. Trümper, The Collatz problem in the light of an infinite free semigroup, Chin. J. Math. 2014, 756917 (2014).

[43] A. Gut, Stopped Random Walks: Limit Theorems and Applications (Springer, New York, 2009).

[44] S. M. Ross, Introduction to Probability Models (Academic, Oxford, 2014).

[45] L. Lacasa and R. Flanagan, Time reversibility from visibility graphs of nonstationary processes, Phys. Rev. E 92, 022817 (2015).

[46] C. L. Faustino, L. R. da Silva, M. G. E. da Luz, E. P. Raposo, and G. M. Viswanathan, Search dynamics at the edge of extinction: Anomalous diffusion as a critical survival state, Europhys. Lett. 77, 30002 (2007).

[47] A. Vespignani and S. Zapperi, How self-organized criticality works: A unified mean-field picture, Phys. Rev. E 57, 6345 (1998).

[48] P. De Los Rios and Y.-C. Zhang, Universal $1 / f$ Noise from Dissipative Self-Organized Criticality, Phys. Rev. Lett. 82, 472 (1999).

[49] V. M. Kenkre, E. W. Montroll, and M. F. Shlesinger, Generalized master equations for continuous-time random walks, J. Stat. Phys. 9, 45 (1973).

[50] B. Drossel, Self-Organized Criticality and Synchronization in a Forest-Fire Model, Phys. Rev. Lett. 76, 936 (1996).

[51] D. L. Turcotte and J. B. Rundle, Self-organized complexity in the physical, biological, and social sciences, Proc. Natl. Acad. Sci. U.S.A. 99, 2463 (2002).

[52] K.-U. Schmidt, Sequence families with low correlation derived from multiplicative and additive characters, IEEE Trans. Inf. Theory 57, 2291 (2011).

[53] W. Ren, A new approach on proving Collatz conjecture, J. Math. 2019, 6129836 (2019).

[54] W. Feller, An Introduction to Probability Theory and its Applications, 3rd ed. (Wiley, Hoboken, 1968), Vol. 1. 